\title{
CXCR4 expression correlates with the degree of tumor infiltration and BRAF status in papillary thyroid carcinomas
}

\author{
Liborio Torregrossa $^{1,4}$, Riccardo Giannini ${ }^{1,4}$, Nicla Borrelli ${ }^{1}$, Elisa Sensi ${ }^{1}$, \\ Rosa Marina Melillo ${ }^{2}$, Pietro Leocata ${ }^{3}$, Gabriele Materazzi ${ }^{1}$, Paolo Miccoli ${ }^{1}$, \\ Massimo Santoro ${ }^{2}$ and Fulvio Basolo ${ }^{1}$ \\ ${ }^{1}$ Dipartimento di Chirurgia, Università di Pisa, Pisa, Italy; ${ }^{2}$ Dipartimento di Biologia e Patologia Cellulare \\ e Molecolare, Università di Napoli Federico II, Napoli, Italy and ${ }^{3}$ Dipartimento di Scienze della Salute, \\ Università dell'Aquila, L'Aquila, Italy
}

\begin{abstract}
Emerging evidence indicates that interactions between chemokine receptors and their ligands may have a critical role in several steps of tumor development, including tumor growth, progression, and metastasis. In this report, we retrospectively evaluated CXCR4 expression in a consecutive series of 200 papillary thyroid carcinomas. We investigated the relationship between the clinicopathological features of the tumors and mutations in the BRAF gene to verify whether overexpression of CXCR4 is linked to more aggressive behavior in thyroid tumors. CXCR4 protein expression was evaluated by immunohistochemical staining. A final staining score was calculated by adding the score representing the percentage of positive cells to the intensity score. The CXCR4 expression of each papillary thyroid carcinoma sample was normalized by calculating the $z$ score for each final staining score. Univariate analysis was used to correlate CXCR4 expression with the papillary thyroid carcinoma variant, the degree of neoplastic infiltration, the American Joint Commission on Cancer stage, the presence of lymphocytic thyroiditis and the mutation status of the BRAF gene. Multiple regression analysis confirmed a strong association between CXCR4, BRAF mutation and the degree of neoplastic infiltration. These data clearly indicate that the chemokine receptor expression induced by oncogenic activation could be the major determinant of the local aggressiveness of neoplastic cells. In conclusion, our data indicate that CXCR4 expression and BRAF mutation status could cooperatively induce and promote a more aggressive phenotype in papillary thyroid carcinoma through several pathways and specifically increase the tumors' spread outside of the thyroid gland.
\end{abstract}

Modern Pathology (2012) 25, 46-55; doi:10.1038/modpathol.2011.140; published online 9 September 2011

Keywords: BRAF; CXCR4; invasiveness; papillary thyroid carcinoma

Thyroid cancer is the most common endocrine malignancy, and its incidence is increasing rapidly worldwide. ${ }^{1}$ The majority of thyroid cancers are papillary thyroid carcinomas, which account for $>80 \%$ of all thyroid malignancies. ${ }^{2}$ Although papillary thyroid carcinoma is usually indolent and curable with surgery, which is often followed by radioiodine treatment, many patients develop

Correspondence: Dr F Basolo, MD, PhD, Department of Surgery, Division of Pathology, Via Roma, 57; 56126-Pisa, Pisa, Italy.

E-mail: f.basolo@med.unipi.it

${ }^{4}$ These authors contributed equally to this work.

Received 20 May 2011; revised 13 July 2011; accepted 18 July 2011; published online 9 September 2011 more aggressive forms of the disease involving the development of local and distant metastasis or tumor recurrence. ${ }^{3,4}$

Chemokines are a superfamily of chemoattractant proteins that induce cytoskeletal rearrangement, firm adhesion to specific cells and directional migration through their interaction with cognate receptors. ${ }^{5}$ These small proteins have been classified into four groups based on the position of their conserved cysteine residues: CXC, CC, C, and CXC3C. ${ }^{6}$ The CXC and CC chemokines interact with the seven-transmembrane G-protein-linked receptors, CXCR and CCR, respectively. Chemokines have a major role in the recruitment of leukocytes to sites of inflammation. ${ }^{7}$ Moreover, chemokines are 
secreted in the tumor microenvironment by both inflammatory cells and tumor cells themselves. ${ }^{8}$ The binding of chemokines to their receptors triggers a cascade of events, including receptor dimerization, tyrosine phosphorylation, recruitment of heterotrimeric $G$ proteins, and activation of the JAK/STAT, PI3-kinase and MAP kinase pathways. ${ }^{5,6}$ Emerging evidence indicates that interactions between chemokine receptors and their ligands may have a critical role in several steps of tumor development, including tumor growth, progression, and metastasis. ${ }^{9,10}$

The chemokine receptor CXCR4 (CXC chemokine receptor 4 , also called fusin) interacts specifically with the chemokine CXCL12 (ckemokine-C-X-C motif-ligand 12) to exert proliferative and chemotactic effects. CXCL12 is constitutively expressed in the lymph nodes, bone marrow, and liver tissue. ${ }^{9}$ The CXCL12/CXCR4 axis is involved in migration and metastatic processes in several human neoplasms, including carcinoma of the breast, prostate, lung, colon, pancreas, and melanoma. ${ }^{9,11-15}$

In this report, we retrospectively evaluated CXCR4 expression in a consecutive series of papillary thyroid carcinomas and investigated its relationship with the clinicopathological features of the disease to verify whether the overexpression of this chemokine receptor is linked to a more aggressive behavior of the thyroid tumors.

\section{Materials and methods}

\section{Tumor Specimens}

From archives of the Department of Surgical Pathology, University of Pisa, we retrospectively retrieved formalin-fixed and paraffin-embedded thyroid specimens from 200 consecutive patients with papillary thyroid carcinoma diagnosed from January 2006 to April 2006. There were 45 males and 155 females aged 14-85 years (mean age $44 \pm 13$ years). All patients were treated and observed at the Institute of Endocrinology at the University of Pisa and underwent a total or near-total thyroidectomy in the Department of Surgery at the same university. Cervical lymphadenectomy was typically performed to treat lymph nodes that appeared abnormal upon intraoperative examination. This study was approved by the Institutional Review Board, and an informed consent was obtained from all patients. Histopathologic review was conducted by two pathologists (FB and LT). The diagnoses were reassessed according to the World Health Organization's classification of thyroid malignancy. ${ }^{16}$ Hematoxylin- and eosin-stained sections were re-examined, taken into account the known risk factors for thyroid cancer, such as tumor size, multifocality, extrathyroidal tumor extension, and the presence or absence of lymph node metastasis. Tumors were staged according to the seventh edition of the tumor-node-metastasis-based staging system recommended by the American Joint Commission on Cancer (AJCC) and the International Union Against Cancer (Union Internationale Contre le Cancer (UICC)). ${ }^{17}$ Moreover, papillary thyroid carcinomas were divided into four groups according to different degrees of tumor infiltration, as described previously: ${ }^{18}$ group A, totally encapsulated tumors; group B, non-encapsulated tumors without thyroid capsule invasion; group $\mathrm{C}$, tumors with thyroid capsule invasion; and group $\mathrm{D}$, tumors with extrathyroidal extension.

\section{Immunohistochemistry}

The immunohistochemical analyses were performed automatically using the Ventana Benchmark immunostaining system (Ventana Medical Systems, Tucson, AZ, USA). Embedded sections (3-5 $\mu \mathrm{m})$ were deparaffinized in xylene, dehydrated through a graded series of alcohols and processed using a diaminobenzidine detection system (Ventana) following the manufacturer's instructions. Any nonspecific staining caused by endogenous biotin was blocked by pre-incubation with the Endogenous Biotin Blocking Kit (Ventana). CXCR4 immunostaining was performed using a rabbit polyclonal antibody generated from an immunogen represented by a synthetic peptide corresponding to the N-terminal amino acids 1-14 of the human CXCR4 protein provided by Abcam (ab 2074; Cambridge, UK) at a dilution of 1:100. The specificity of the CXCR4 antibody was assessed using a specific blocking peptide (Abcam), following the manufacturer's instructions. To validate the reliability of CXCR4 immunostaining with the Abcam primary antibody, we performed immunohistochemical analysis using another rabbit polyclonal antibody raised against amino acids 176-293 of fusin provided by Santa Cruz Biotechnology (Santa Cruz, CA, USA) diluted $1: 100$. Analysis with the second antibody was performed in a subgroup of 40 papillary thyroid carcinoma samples. Diaminobenzidine was used as a chromogen, and commercial hematoxylin was used for counterstaining. Positive controls were always used and prepared using samples of colon epithelium following the manufacturer's instruction. For the negative control, all of the reagents described above were used without the inclusion of the primary antibody.

\section{Assessment of Immunoreactivity}

At least 10 representative fields under high-power magnification $(\times 40)$ were chosen. The cellular localization of the immunostaining was recorded. CXCR4 immunoreactivity was independently evaluated by two pathologists (LT and FB) who were blinded to the clinicopathological data. Different scores between the two investigators were observed in $<10 \%$ of the cases, and a consensus was achieved in all cases after discussion. Semiquanti- 
tative scores were given as the score of the percentage of positive cells plus the score of the staining intensity. To score the samples based on the percentage of positive cells, the following criteria were used: samples with $0-5 \%$ positive neoplastic cells were given a score of 0 ; samples with $6-25 \%$ positive neoplastic cells were given a score of 1 ; samples with $26-50 \%$ positive neoplastic cells were given a score of 2; samples with $51-75 \%$ positive neoplastic cells were given a score of 3 ; and samples with $76-100 \%$ positive neoplastic cells were given a score of 4 . Samples were classified as positive if $>5 \%$ of the neoplastic cells showed specific immunoreactivity; otherwise, they were classified as negative. The intensity score was given as follows: samples with no staining were given a score of 0 ; samples with weak/equivocal staining were given a score of 1 ; samples with mild staining were given a score of 2; samples with moderate staining were given a score of 3 ; and samples with strong staining were given a score of 4 . A final staining score was calculated by adding the score corresponding to the percentage of positive cells and the intensity score, and the scores ranged from 0 to 8. The CXCR4 expression of each papillary thyroid carcinoma sample was normalized by calculating the $z$ score for each final staining score. The $z$ score normalization functions to equalize the variability of the immunohistochemical staining among the different samples. Every final staining score was converted to a $z$ score by subtracting the tumor cell population mean and dividing by the s.d. Thus, a positive $z$ score indicates a value above the population mean and a negative score indicates a value below the mean. The converted $z$ scores were then aggregated into one large set, identifying two different categories: 'low expression' corresponding to a negative $z$ score or 'high expression' corresponding to a positive $z$ score.

\section{RNA Isolation and Complementary DNA (cDNA) Synthesis}

Total cellular RNA was isolated from formalin-fixed and paraffin-embedded (FFPE) thyroid tissue using the RNAeasy FFPE Kit (Qiagen) in accordance with the manufacturer's instructions. In all, $500 \mathrm{ng}$ of total RNA were used to synthesize cDNA using the RevertAid First Strand cDNA Synthesis Kit (Fermentas) in a reaction volume of $20 \mu \mathrm{l}$.

\section{Semiquantitative Real-Time RT-PCR}

The quantification of CXCR4 mRNA was performed using the two-step real-time quantitative RT-PCR Rotor Gene Sybr Green PCR Kit (Qiagen) on a Rotor Gene 6000 (Qiagen) instrument. Quanti Tect Primer Assay (Qiagen) for CXCR4 (NM_003467) and $\beta$-Actin (NM_001101) was used as an internal control. Additionally, $2.5 \mu \mathrm{l}$ of cDNA were used in a final volume of $25 \mu \mathrm{l}$. Amplification conditions were in accordance with the manufacturer's instructions. The comparative threshold cycle (Ct) method, defined as $2^{-\Delta \Delta \mathrm{Ct}}$, was used for the calculation of fold amplification. Each experiment was evaluated with three PCR reactions. Data are presented as the mean value \pm s.d.

\section{BRAF Mutation Analysis}

Detection of $B R A F$ mutations was performed according to the standard procedures using PCR-Single Strand Conformation Polymorphism and direct DNA sequencing. ${ }^{19}$

\section{Statistical Analysis}

We performed univariate and multivariate analyses using Statistica Software (Stat Soft). We used a nonparametric $\chi^{2}$ test to investigate the differences between the variables of the different groups. $P$-values were determined at $95 \%$ confidence intervals. The values of the odds ratio were used to study the association between different groups. Next, we proceeded with a multiple regression analysis to highlight how CXCR4 expression influenced the different variables in a system with multiple interactions.

\section{Results}

\section{Pathology Review}

In total, $68(34 \%)$ cases were histologically diagnosed as microcarcinoma (defined as a tumor with a diameter of $10 \mathrm{~mm}$ or less), $39(21 \%)$ as the follicular variant, $83(42 \%)$ as the classic variant, and $10(5 \%)$ as the tall cell variant of papillary thyroid carcinoma. The mean tumor size was $1.39 \pm 0.78 \mathrm{~cm}$ (ranging from 0.5 to $7 \mathrm{~cm}$ ). Tumor multifocality was observed in 47 cases (23\%), and lymph node metastasis was identified in 55 cases $(27 \%)$. According to the degree of neoplastic infiltration, papillary thyroid carcinomas were subdivided into the following groups: group A included $55(27 \%)$ totally encapsulated tumors; group B included 45 (23\%) non-encapsulated tumors without thyroid capsule invasion; group C included $30(15 \%)$ tumors with thyroid capsule invasion; and group D included 70 $(35 \%)$ tumors with extrathyroidal invasion. In line with the AJCC/UICC tumor-node-metastasis-based staging system, 169 patients $(84 \%)$ were staged as I, $5(3 \%)$ as II, $18(9 \%)$ as III, and $8(4 \%)$ as IVa. The presence of lymphocytic thyroiditis was observed in $72(36 \%)$ cases (Table 1$)$. 
Table 1 Correlation between CXCR4 immunohistochemical expression and clinicopathological features

\begin{tabular}{|c|c|c|c|c|c|c|}
\hline \multirow[t]{2}{*}{ Clinical-pathological features } & \multicolumn{2}{|c|}{ CXCR4 FSS } & \multicolumn{4}{|c|}{ CXCR4 z score } \\
\hline & Mean FSS & $\mathrm{P}$ & High n (\%) & Low n $(\%)$ & $\mathrm{P}$ & OR $(95 \% C I)$ \\
\hline \multicolumn{7}{|l|}{ Age at diagnosis } \\
\hline Patients $<45$ years $(n=110)$ & $3.36 \pm 2.3$ & 0.5 & $33(30.0)$ & $77(70.0)$ & 0.072 & $0.59(0.33-1.05)$ \\
\hline Patients $\geqslant 45$ years $(n=90)$ & $3.53 \pm 2.5$ & & $38(42.2)$ & $52(57.8)$ & & \\
\hline \multicolumn{7}{|l|}{ Gender } \\
\hline Male $(n=45)$ & $3.73 \pm 2.5$ & 0.38 & $17(37.8)$ & $28(62.2)$ & 0.72 & $1.14(0.57-2.26)$ \\
\hline Female $(n=155)$ & $3.35 \pm 2.4$ & & $54(34.8)$ & $101(65.2)$ & & \\
\hline \multicolumn{7}{|l|}{ PTC variants } \\
\hline MicroPTC $(n=68)$ & $3.79 \pm 2.6$ & 0.00005 & $29(42.6)$ & $39(57.4)$ & & \\
\hline $\mathrm{FV}(n=39)$ & $1.74 \pm 1.6$ & & $2(5.0)$ & $37(95.0)$ & $<0.00005$ & $\mathrm{~b}$ \\
\hline $\mathrm{CV}(n=83)$ & $3.66 \pm 2.2$ & & $32(38.5)$ & $51(61.5)$ & & \\
\hline $\operatorname{TCV}(n=10)$ & $5.80 \pm 1.6$ & & $8(80.0)$ & $2(20.0)$ & & \\
\hline \multicolumn{7}{|l|}{ Multifocality $^{\mathrm{a}}$} \\
\hline No $(n=153)$ & $3.50 \pm 2.4$ & 0.62 & $53(34.6)$ & $100(65.4)$ & 0.64 & $1.17(0.60-2.30)$ \\
\hline Yes $(n=47)$ & $3.23 \pm 2.4$ & & $18(38.3)$ & $29(61.7)$ & & \\
\hline \multicolumn{7}{|l|}{ Lymph node metastasis } \\
\hline No $(n=145)$ & $3.18 \pm 2.5$ & 0.019 & $46(31.7)$ & $99(68.3)$ & 0.07 & $1.79(0.95 \mathrm{v} 3.39)$ \\
\hline Yes $(n=55)$ & $4.10 \pm 2.1$ & & $25(45.5)$ & $30(54.5)$ & & \\
\hline \multicolumn{7}{|l|}{ Degree of neoplastic infiltration } \\
\hline $\mathrm{A}(n=55)$ & $2.18 \pm 2.0$ & 0.00005 & 8 (14.5) & $47(85.5)$ & & \\
\hline $\mathrm{B}(n=45)$ & $2.93 \pm 2.7$ & & $11(24.4)$ & $34(75.6)$ & $<0.00005$ & b \\
\hline $\mathrm{C}(n=30)$ & $4.16 \pm 2.3$ & & $15(50.0)$ & $15(50.0)$ & & \\
\hline $\mathrm{D}(n=70)$ & $4.44 \pm 2.0$ & & $37(52.8)$ & $33(47.2)$ & & \\
\hline \multicolumn{7}{|l|}{ AJCC stages } \\
\hline $\mathrm{I}(n=169)$ & $3.31 \pm 2.4$ & 0.04 & $53(31.4)$ & $116(68.6)$ & & \\
\hline II $(n=5)$ & $1.80 \pm 2.6$ & & $1(20.0)$ & $4(80.0)$ & 0.063 & b \\
\hline III $(n=18)$ & $4.55 \pm 2.2$ & & $12(66.7)$ & $6(33.3)$ & & \\
\hline IVa $(n=8)$ & $4.50 \pm 2.4$ & & $5(62.5)$ & $3(37.5)$ & & \\
\hline \multicolumn{7}{|l|}{ Lymphocytic thyroiditis } \\
\hline No $(n=128)$ & $3.13 \pm 2.5$ & 0.022 & $38(29.7)$ & $90(70.3)$ & 0.022 & $2.00(1.10-3.65)$ \\
\hline Yes $(n=72)$ & $3.98 \pm 2.2$ & & $33(45.8)$ & $39(54.2)$ & & \\
\hline \multicolumn{7}{|l|}{ BRAF status } \\
\hline Wild type $(n=110)$ & $2.40 \pm 2.2$ & 0.00005 & $21(19.1)$ & $89(80.9)$ & $<0.00005$ & $5.30(2.82-9.96)$ \\
\hline V600E mutated $(n=90)$ & $4.70 \pm 2.0$ & & $50(55.5)$ & $40(44.5)$ & & \\
\hline
\end{tabular}

Abbreviations: AJCC, American Joint Commission on Cancer; CI, confidence interval; CV, classical variant of PTC; FV, follicular variant of PTC; FSS, final staining score; OR, odds ratio; PTC, papillary thyroid carcinoma; TCV, tall cell variant of PTC.

${ }^{\mathrm{a}}$ The neoplasm with the greatest size or the highest pT status has always been analyzed in the presence of multifocality.

${ }^{\mathrm{b}}$ Not done.

\section{CXCR4 Protein Expression by Immunohistochemistry}

As previously observed, the two CXCR4 antibodies yield similar results: no difference was noted between the anti-CXCR4 antibodies provided either by Abcam or by Santa Cruz. Furthermore, blocking experiments with corresponding immunizing peptide confirmed the specificity of the antibodies. ${ }^{20}$ CXCR4 immunohistochemical expression was assessed in each tumor and in the adjacent nonneoplastic parenchyma. CXCR4 showed a cytoplasmic pattern of immunoreactivity with occasional perinuclear ('Golgi-like') staining. According to the final staining score, CXCR4 expression was observed in 160 out of $200(80 \%)$ cases. The remaining cases ( 40 out of $200,20 \%$ ) were considered negative (that is, final staining score $=0$ ). Among these, 11 $(27.5 \%)$ were diagnosed as classic variants, 16 $(40 \%)$ as follicular variants, and $13(32.5 \%)$ as microcarcinomas. No tall cell variants were observed in this group. Overall, the mean final staining score was $3.44 \pm 2.4$. Considering only the papillary thyroid carcinomas expressing CXCR4, the mean final staining score was $4.3 \pm 1.9$. Based on the calculated $z$ score, $71(35.5 \%)$ papillary thyroid carcinomas showed high expression of CXCR4, and $129(64.5 \%)$ showed low expression. Examples of tumors scored as having positive CXCR4 expression are shown in Figures 1 and 2. Focusing on adjacent non-neoplastic parenchyma, CXCR4 protein was 

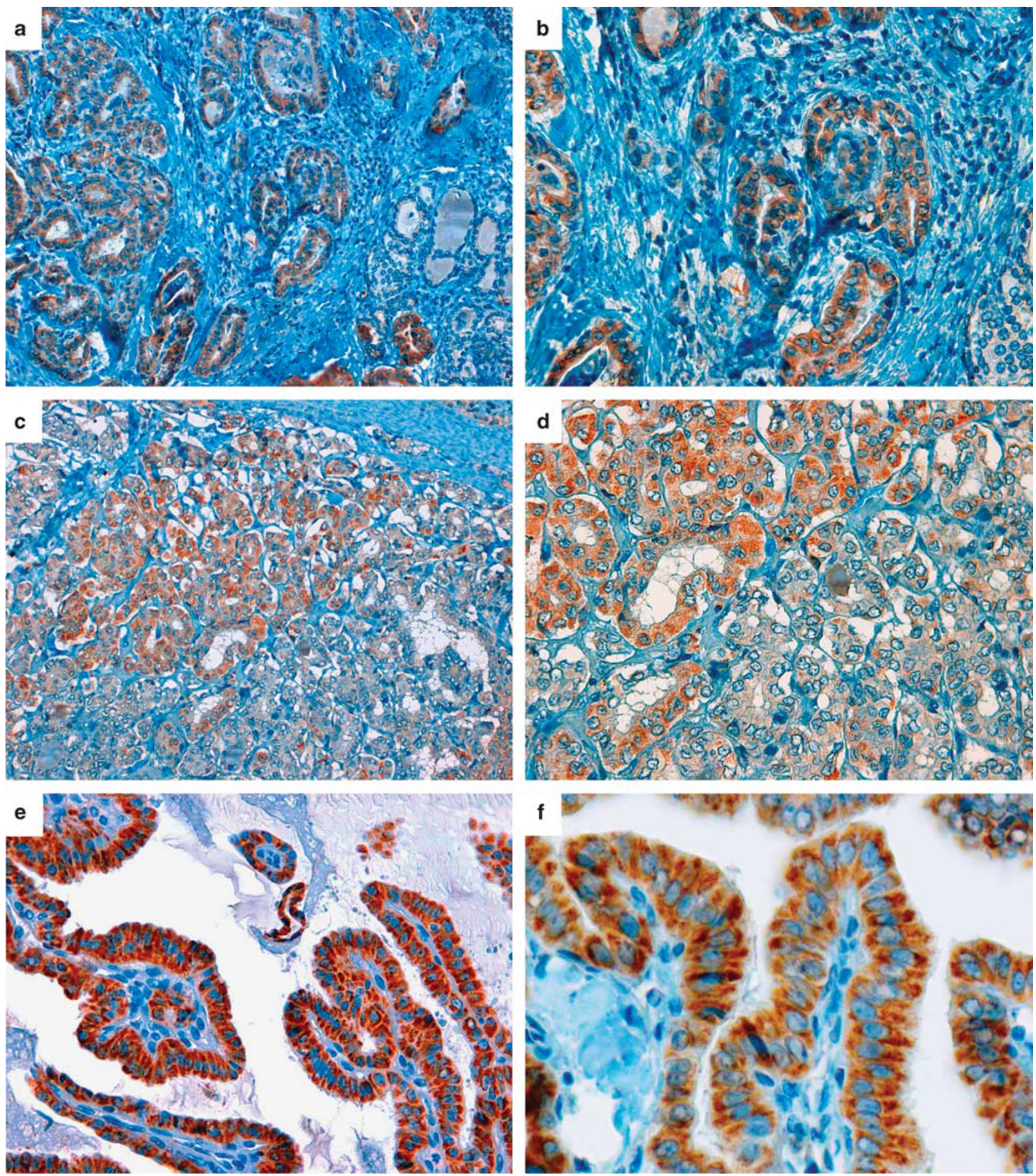

Figure 1 CXCR4 immunohistochemical staining of papillary thyroid carcinoma variants. (a, b) Microcarcinoma showing a final staining score $=7, \times 20$ and $\times 40 ;(\mathbf{c}, \mathbf{d})$ follicular variant of papillary thyroid carcinoma showing a final staining score $=4, \times 20$ and $\times 40 ;(\mathbf{e}, \mathbf{f})$ classic variant of papillary thyroid carcinoma showing a final staining score $=8$, magnification $\times 20$ and $\times 40$. Each pair of immunohistochemical stains is from the same sample at different magnifications.

variably expressed in the cytoplasm of follicular cells with oxyphilic metaplasia in areas with lymphocytic thyroiditis and in some types of cells related to inflammation (ie, macrophages), as described previously. ${ }^{20}$ Normal thyroid tissue neighboring the neoplasm was consistently negative.

\section{BRAF Mutational Status}

$B R A F$ mutations were found in 90 of 200 papillary thyroid carcinoma cases $(45 \%)$. Specifically, we found only the BRAF V600E transversion. With respect to the papillary thyroid carcinoma variant, 
BRAF V600E was present in $43.5 \%$ of the microcarcinomas (30 of 69 ), in $17.9 \%$ of the follicular variants (7 of 39), in $56.1 \%$ of the classic variants (46 of 82 ), and in $70.0 \%$ of the tall cell variants (7 of 10 ).

\section{CXCR4 mRNA Expression by Real-Time qRT-PCR}

CXCR4 mRNA levels were assessed in a subset of tumors using semiquantitative RT-PCR with primers specific for CXCR4. CXCR4 mRNA expression was evaluated based on an internal reference gene to determine the fold-difference in CXCR4 mRNA levels in tumor samples vs CXCR4 mRNA levels in a pool of normal thyroid samples; the latter was negative by immunohistochemical evaluation. The median CXCR4 mRNA expression was 0.19, 0.39, $0.802,0.64$, and 1.42 for representative samples with an immunohistochemical final staining score of 0,2 , 6,7 and 8 , respectively (Spearman's coefficient $r s=0.37 ; P=0.022$ ).
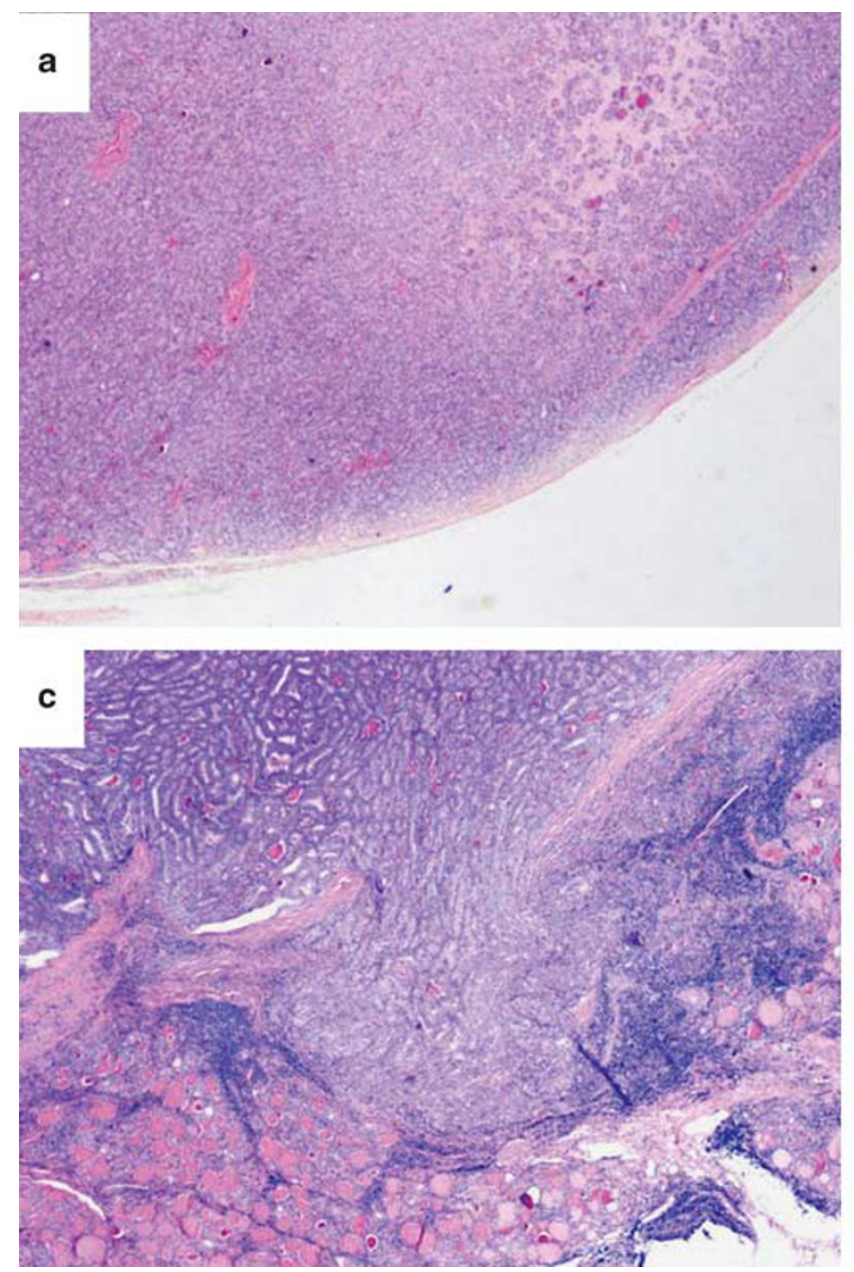

\section{Correlation Between CXCR4 Protein Expression by Immunohistochemistry, Clinicopathological Features and BRAF Mutational Status}

The tumor size inversely correlated with the final staining score $(r=-0.25 ; P<0.005)$. This correlation was confirmed by $z$ score analysis: the mean size of the tumors expressing low levels of CXCR4 was significantly different from the mean size of the tumors expressing high levels of CXCR4 $(1.5 \pm 0.9 \mathrm{~cm}$ and $1.2 \pm 0.4 \mathrm{~cm}$, respectively, $P=0.02$; Figure 3 ). By univariate analysis, no significant association between age at diagnosis, gender, multifocality, and CXCR4 protein expression was observed in terms of both mean final staining score and $z$ score (Table 1).

Conversely, a significant correlation was observed between CXCR4 expression and the papillary thyroid carcinoma variant based on the Kruskall-Wallis non-parametric test considering both final staining score $(P=0.00005)$ and $z$ score $(P<0.00005)$. The mean final staining score was $3.79 \pm 2.6$ in micro-
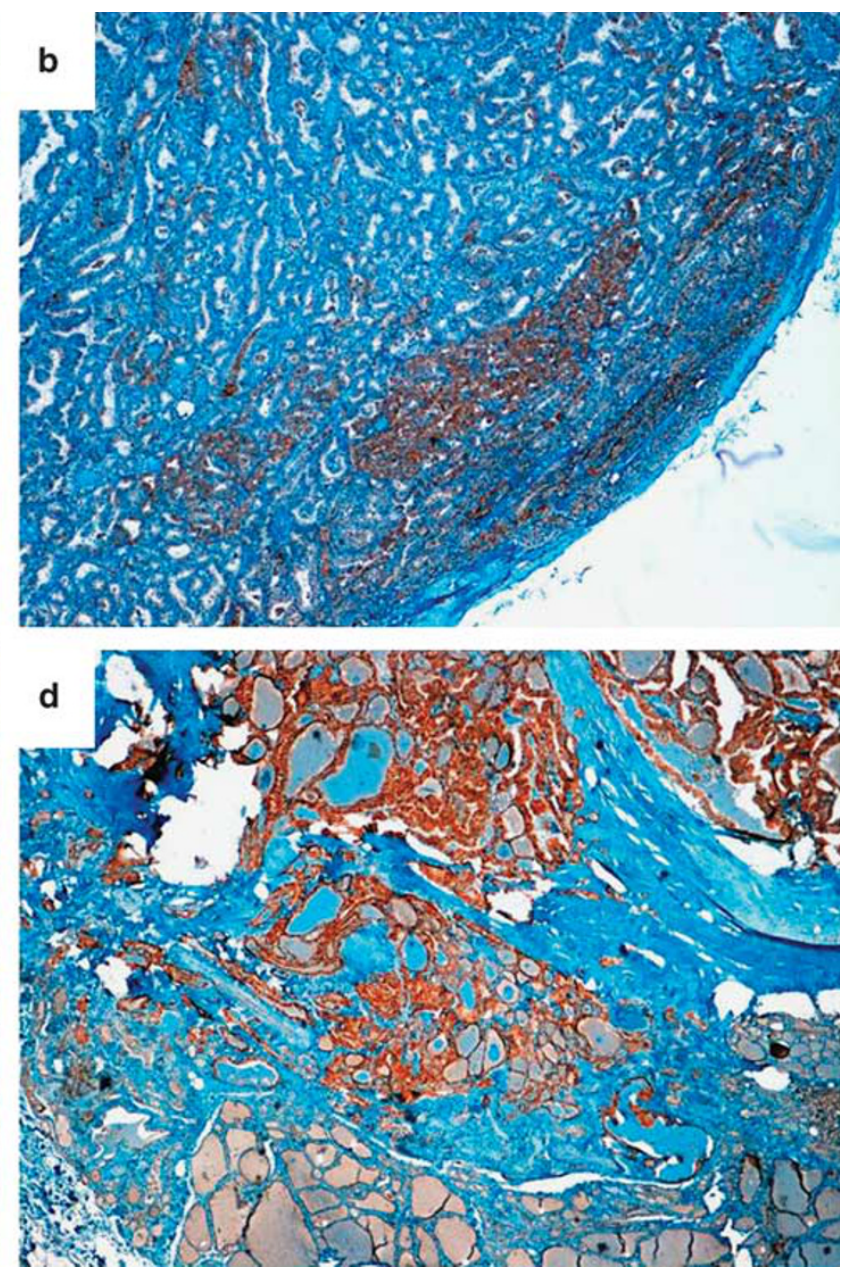

Figure 2 Hematoxylin- and eosin-stained sections representing the four degrees of neoplastic infiltration and CXCR4 immunohistochemical staining $(\times 20$ magnification). (a, b) Fully encapsulated papillary thyroid carcinoma with an final staining score $=4 ;(\mathbf{c}, \mathbf{d})$ nonencapsulated papillary thyroid carcinoma without thyroid capsule invasion with an final staining score $=7$; (e, f) papillary thyroid carcinoma with thyroid capsule invasion with an final staining score =7; (g, h) papillary thyroid carcinoma with extrathyroidal extension with an final staining score $=7$. Each pair of hematoxylin- and eosin-stained slides and immunohistochemical stains is from the same sample. Whole arrow indicates thyroid capsule; asterisk indicates soft perithyroidal tissues. 

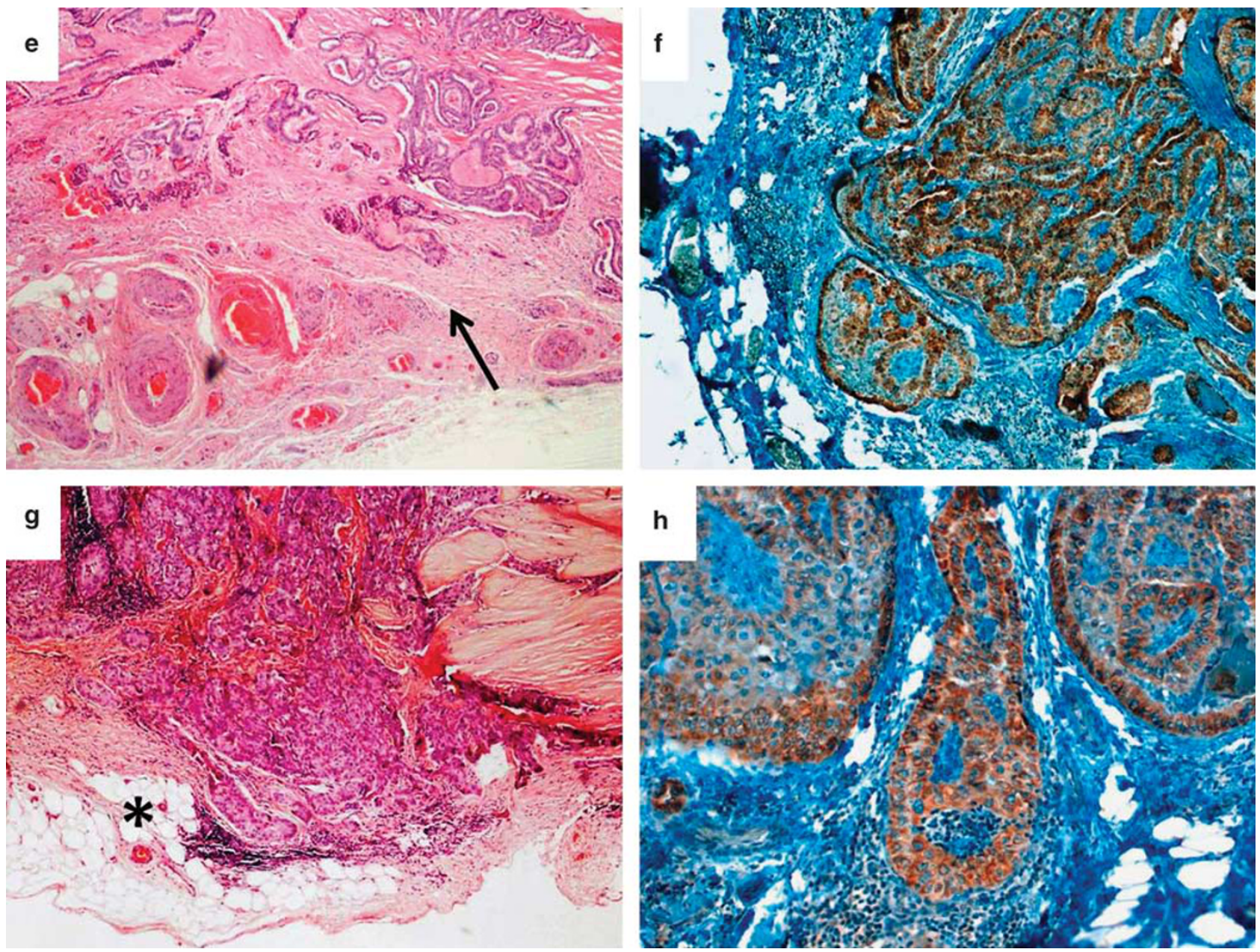

Figure 2 Continued.

carcinomas, $1.74 \pm 1.6$ in follicular variants, $3.66 \pm 2.2$ in classic variants, and $5.80 \pm 1.6$ in tall cell variants. In terms of $z$ score, high levels of CXCR4 expression were observed in 29 (42.6\%) microcarcinomas, $2(5 \%)$ follicular variants, 32 $(38.5 \%)$ classic variants, and $8(80 \%)$ tall cell variants (Figure 4a).

The lymph node metastatic tumors showed a mean final staining score significantly higher than the non-metastatic tumors $(4.10 \pm 2.1$ and $3.18 \pm 2.5$, respectively, $P=0.019$ ). Nevertheless, no significant association was found using the $z$ score, although the $P$ value is very close to significant $(P=0.07$; $\mathrm{OR}=1.79$; Figure $4 \mathrm{~b}$ ).

Statistical analysis showed significant correlation between CXCR4 expression and the degree of neoplastic infiltration based on the Kruskall-Wallis non-parametric test considering both final staining score $(P=0.00005)$ and $z$ score $(P<0.00005)$. The mean final staining score was $2.18 \pm 2.0$ in group A, $2.93 \pm 2.7$ in group $\mathrm{B}, 4.16 \pm 2.3$ in group $\mathrm{C}$, and $4.44 \pm 2.0$ in group D; by z score, $8(14.5 \%)$ cases in group A, $11(24.4 \%)$ cases in group B, $15(50.0 \%)$

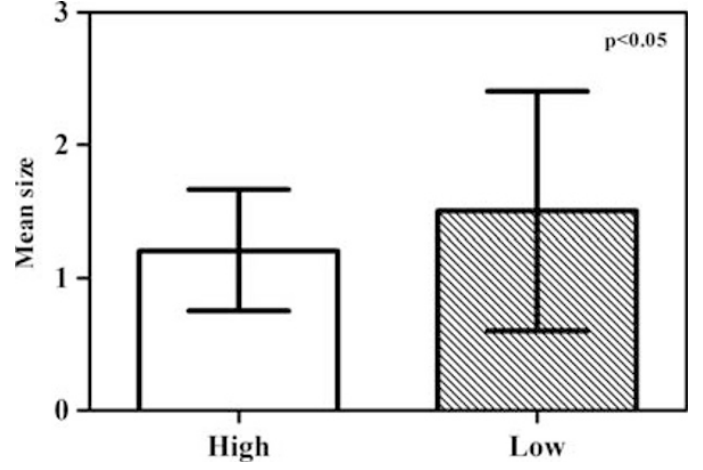

Figure 3 Correlation between CXCR4 level of expression ( $z$ score) and mean tumor size (bars represent the s.d.s).

cases in group C, and $37(52.8 \%)$ cases in group D showed high levels of CXCR4 (Figure 4c). Based on the Kruskall-Wallis non-parametric test, CXCR4 expression was also significantly correlated to AJCC stage in terms of final staining score $(P=0.04)$. The mean final staining score was $3.31 \pm 2.4,1.80 \pm 2.6$, 

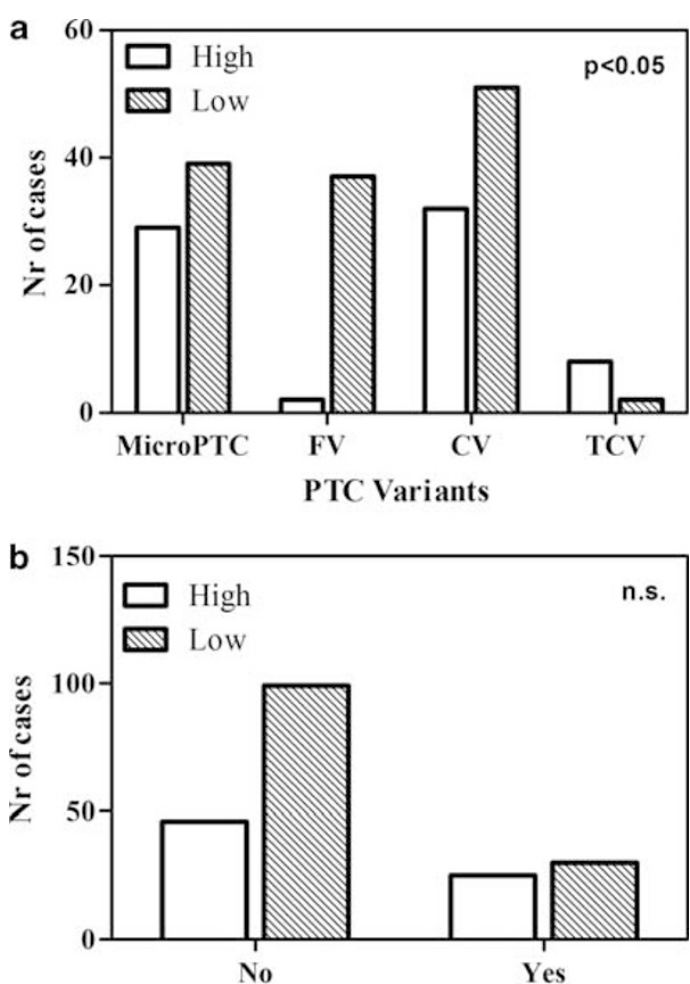

Presence of lymphnode metastases

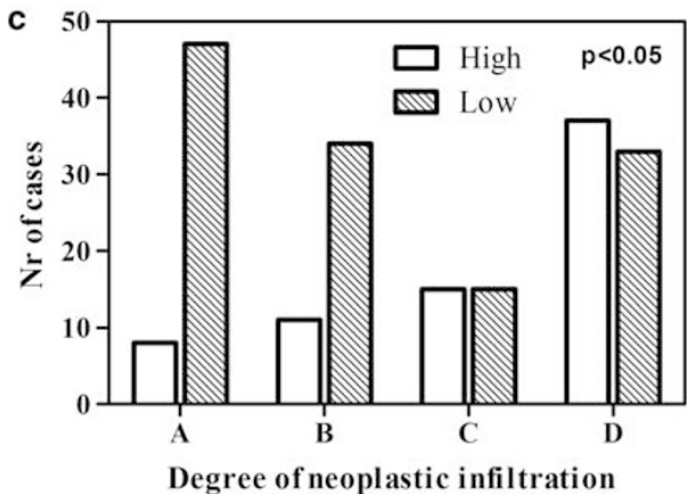

Figure 4 Correlation between the CXCR4 level of expression ( $z$ score) and: (a) papillary thyroid carcinoma variants; (b) presence of lymph node metastasis; (c) degree of neoplastic infiltration.

$4.55 \pm 2.2$, and $4.50 \pm 2.4$ for tumors of stage I, II, III, and IVa, respectively.

The mean final staining score of papillary thyroid carcinomas associated with lymphocytic thyroiditis $(3.98 \pm 2.2)$ was significantly higher $(P=0.022)$ than the mean of papillary thyroid carcinomas without lymphocytic thyroiditis $(3.13 \pm 2.5)$. These data were also confirmed by $z$ score: high levels of protein expression were statistically associated with lymphocytic thyroiditis $(P=0.022$; $\mathrm{OR}=2.00)$.

Interestingly, the mean final staining score was significantly higher $(P=0.00005)$ in BRAF V600Emutated tumors $(4.7 \pm 2.0)$ compared with $B R A F$ wild-type tumors $(2.4 \pm 2.2)$; in addition, mutated tumors expressed levels of CXCR4 that were sig-
Table 2 Multiple regression analysis of the association between CXCR4 immunohistochemical expression and clinicopathological features

\begin{tabular}{lcc}
\hline Clinical-pathological features & $\begin{array}{c}\text { CXCR4 FSS } \\
\text { (P value) }\end{array}$ & $\begin{array}{c}\text { CXCR4 z score } \\
\text { (P value) }\end{array}$ \\
\hline PTC variants & 0.0181 & $\mathrm{NS}$ \\
Lymph node metastasis & 0.0310 & $\mathrm{a}$ \\
Degree of neoplastic infiltration & 0.0015 & 0.0173 \\
AJCC stages & $\mathrm{NS}$ & $\mathrm{a}$ \\
Lymphocytic thyroiditis & $\mathrm{NS}$ & $\mathrm{NS}$ \\
$B R A F$ status & 0.0000 & 0.0002 \\
\hline
\end{tabular}

Abbreviations: AJCC, American Joint Commission on Cancer; FSS, final staining score; NS, not significant; PTC, papillary thyroid carcinoma.

${ }^{\mathrm{a}}$ Not significant in univariate analysis.

nificantly higher than non-mutated tumors $(P<0.00005 ; \mathrm{OR}=5.30)$ according to the $z$ scores.

By multiple linear regression analysis, papillary thyroid carcinoma variants, lymph node metastasis, degree of neoplastic infiltration, and $B R A F$ status were statistically associated with CXCR4 final staining score. Alternatively, considering the $z$ scores, the statistical association remained significant only for the degree of neoplastic infiltration and BRAF status (Table 2).

\section{Discussion}

CXCR4 expression has been reported in RET/PTCpositive human thyroid cancer cell lines and in papillary thyroid carcinoma samples, but not in normal thyroid cells. ${ }^{20-24}$ In addition, we demonstrated that CXCR4, in association with HBME-1 protein expression, was significantly higher in papillary thyroid carcinoma than in benign lesions. ${ }^{20}$ Hence, these proteins have been proposed as markers in the differential diagnosis of malignant vs benign thyroid nodules.

In this study, we analyzed CXCR4 expression in a consecutive series of two hundred papillary thyroid carcinomas focusing on the correlation between CXCR4 and clinicopathological features, such as tumor size, age, gender, histological variant, multifocality, presence of node metastases, degree of neoplastic infiltration, AJCC stage, and the presence of lymphocytic thyroiditis. Because BRAF mutation is one of the most important gene alterations found in papillary thyroid carcinoma, we also correlated this oncogene activation with expression of CXCR4.

In our series, CXCR4 expression correlated with papillary thyroid carcinoma variant. Specifically, high CXCR4 expression was found in the tall cell and classic variants, whereas low levels were observed in the follicular variant, as reported previously. ${ }^{20,25}$ Moreover, the inverse correlation observed between tumor size and CXCR4 expression suggests that the papillary thyroid carcinoma var- 
iant type is more important for modulating CXCR4 expression than tumor size. These results are in contrast with the data previously reported by Wagner et $a{ }^{26}{ }^{26}$ showing a correlation between CXCR4 expression and larger tumor size.

In our samples, we found a strong correlation between CXCR4 expression and the degree of neoplastic infiltration. More specifically, we found a high level of CXCR4 expression not only in association with extrathyroidal extension but also corresponding to thyroid capsule infiltration. Our data are in contrast to studies reporting a lack of correlation between CXCR4 expression and extrathyroidal extension of the tumor. ${ }^{25,26}$ We can speculate that tumor infiltration of the thyroid capsule may represent a crucial step in local invasion, and this process may be modulated by CXCR4 expression.

AJCC stage correlates with CXCR4 expression only in terms of final staining score. Despite the lack of statistical significance with respect to the $z$ score, it is clear that the percentage of stage III and IVa tumors expressing high levels of CXCR4 is almost twice that observed in stage I tumors. The correlation between CXCR4 expression and higher tumor stage was partially confirmed by the results regarding lymph node metastatic papillary thyroid carcinomas. In fact, the presence of lymph node metastases correlates with CXCR4 expression in terms of final staining score. Our findings confirm CXCR4 involvement in lymphatic tumor metastasis. ${ }^{25,27}$

In line with previously reported associations found between inflammation and CXCR4 expression by malignant epithelial cells, ${ }^{21}$ we found that lymphocytic thyroiditis correlates with expression of this receptor. Nevertheless, others have reported the lack of correlation between chemokine production and chemokine receptor expression in papillary thyroid carcinoma cells and the degree of inflammatory infiltrate in the thyroid. ${ }^{26}$ One of the possible reasons for this discrepancy may be because of the different numbers of cases evaluated; in the report, we studied more than twice the number of tumors as Wagner's study. ${ }^{26}$ However, further evaluation may be necessary to clarify this point.

In addition, our findings show that the $B R A F$ V600E gene mutation strongly correlates with high levels of CXCR4 expression. Melillo et al ${ }^{24}$ proposed that activation of the RET/PTC-RAS-BRAF axis results in upregulation of chemokines and their receptors, which are relevant for sustained proliferation and cell motility of papillary thyroid carcinoma tumor cells. The authors speculated that these genes may act cooperatively to commit transformed thyroid cells to a malignant, invasive phenotype. To verify whether CXCR4 expression depends on RAS or BRAF activation, Castellone et $a l^{22}$ examined CXCR4 mRNA levels in two cell lines, which expressed the Ha-RAS V12 and BRAF V600E oncogenes, respectively, and found CXCR4 upregulation in both cell lines. Borrello et $a l^{21}$ reported that the RET/PTC1 oncogene induces the expression of a large set of genes involved in inflammation and tumor invasion, including those encoding chemokines (eg, CXCL12), chemokine receptors (CXCR4), cytokines, matrix-degrading enzymes, and adhesion molecules. In the same study, they demonstrated that CXCR4-positive thyrocytes migrate in response to CXCL12, suggesting that the CXCR4/CXCL12 axis may be involved in the metastatic capacity of tumor cells. Taken together, the above mentioned results suggest that CXCR4 expression in papillary thyroid carcinoma can be upregulated by $B R A F$ activation, leading to more aggressive behavior of the tumor in terms of local invasion and lymphatic spread.

Multiple regression analysis confirms the strong association between CXCR4, BRAF and the degree of neoplastic infiltration. These data clearly indicate that the chemokine receptor expression induced by oncogenic activation is the major determinant of the local spread of the neoplastic cells. These observations are in line with our previous paper demonstrating a strong association between BRAF mutation and tumor invasiveness. ${ }^{18}$

In conclusion, our data indicate that CXCR4 expression and BRAF mutation status could cooperatively induce and promote a more aggressive papillary thyroid carcinoma phenotype and specifically increase tumor spread outside the thyroid gland.

\section{Acknowledgements}

This work was supported by the Associazione Italiana per la Ricerca sul Cancro (Italy) and by the COFIN grant titled 'Characterization of tumour inflammatory infiltrate in papillary thyroid carcinomas' no. 2007MBW5M7_004 from the Italian Minister of University and Scientific Research.

\section{Disclosure/conflict of interest}

The authors declare no conflict of interest.

\section{References}

1 Enewold L, Zhu K, Ron E, et al. Rising thyroid cancer incidence in the United States by demographic and tumor characteristics, 1980-2005. Cancer Epidemiol Biomarkers Prev 2009;18:784-791.

2 Nikiforov YE. Thyroid carcinoma: molecular pathways and therapeutic targets. Mod Pathol 2008;21(Suppl 2): S37-S43.

3 Mazzaferri EL, Jhiang SM. Long-term impact of initial surgical and medical therapy on papillary and follicular thyroid cancer. Am J Med 1994;97:418-428. 
4 Sherman SI, Brierley JD, Sperling M, et al. Prospective Multicenter Study of Thyrois Carcinoma Treatment: Initial Analysis of Staging and Outcome. National Thyroid Cancer Treatment Cooperative Study Registry Group. Cancer 1998;83:1012-1021.

5 Ganju RK, Brubaker SA, Meyer J, et al. The alphachemokine, stromal cell-derived factor-1alpha, binds to the transmembrane G-protein-coupled CXCR-4 receptor and activates multiple signal transduction pathways. J Biol Chem 1998;273:23169-23175.

6 Payne AS, Cornelius LA. The role of chemokines in melanoma tumor growth and metastasis. J Invest Dermatol 2002;118:915-922.

7 Luster AD. Chemokines-chemotactic cytokines that mediate inflammation. N Engl J Med 1998;338:436-445.

8 Bozec A, Lassalle S, Hofman V, et al. The thyroid gland: a crossroad in inflammation-induced carcinoma? An ongoing debate with new therapeutic potential. Curr Med Chem 2010;17:3449-3461.

9 Muller A, Homey B, Soto $\mathrm{H}$, et al. Involvement of chemokine receptors in breast cancer metastasis. Nature 2001;410:50-56.

10 Smith MC, Luker KE, Garbow JR, et al. CXCR4 regulates growth of both primary and metastatic breast cancer. Cancer Res 2004;64:8604-8612.

11 Taichman RS, Cooper C, Keller ET, et al. Use of the stromal cell-derived factor-1/CXCR4 pathway in prostate cancer metastasis to bone. Cancer Res 2002; 62:1832-1837.

12 Burger M, Glodek A, Hartmann T, et al. Functional expression of CXCR4 (CD184) on small-cell lung cancer cells mediates migration, integrin activation, and adhesion to stromal cells. Oncogene 2003;22: 8093-8101.

13 Kim J, Takeuchi H, Lam ST, et al. Chemokine receptor CXCR4 expression in colorectal cancer patients increases the risk for recurrence and for poor survival. J Clin Oncol 2005;23:2744-2753.

14 Saur D, Seidler B, Schneider G, et al. CXCR4 expression increases liver and lung metastasis in a mouse model of pancreatic cancer. Gastroenterology 2005; 129:1237-1250.

15 Bartolome RA, Ferreiro S, Miquilena-Colina ME, et al. The chemokine receptor CXCR4 and the metalloproteinase MT1-MMP are mutually required during melanoma metastasis to lungs. Am J Pathol 2009; 174:602-612.

16 LiVolsi VA, Albores-Saavedra J, Asa SL, et al. Papillary carcinoma. In: DeLellis RA, Lloyd RV, Heitz PU, Eng C, (eds). World Health Organization Classifica- tion of Tumours. Pathology and Genetics of Tumours of Endocrine organs. IARC Press, Lyon, 2004, pp 57-66.

17 Sobin LHGM, Wittekind Ch, (ed). TNM Classification of Malignant Tumors. Wiley-Blackwell, Oxford, 2009, $310 \mathrm{pp}$.

18 Basolo F, Torregrossa L, Giannini R, et al. Correlation between the BRAF V600E mutation and tumor invasiveness in papillary thyroid carcinomas smaller than 20 millimeters: analysis of 1060 cases. J Clin Endocrinol Metab 2010;95:4197-4205.

19 Lupi C, Giannini R, Ugolini C, et al. Association of BRAF V600E mutation with poor clinicopathological outcomes in 500 consecutive cases of papillary thyroid carcinoma. J Clin Endocrinol Metab 2007;92: 4085-4090.

20 Torregrossa L, Faviana $\mathrm{P}$, Filice $\mathrm{ME}$, et al. CXC chemokine receptor 4 immunodetection in the follicular variant of papillary thyroid carcinoma: comparison to galectin-3 and hector battifora mesothelial cell-1. Thyroid 2010;20:495-504.

21 Borrello MG, Alberti L, Fischer A, et al. Induction of a proinflammatory program in normal human thyrocytes by the RET/PTC1 oncogene. Proc Natl Acad Sci USA 2005;102:14825-14830.

22 Castellone MD, Guarino V, De Falco V, et al. Functional expression of the CXCR4 chemokine receptor is induced by RET/PTC oncogenes and is a common event in human papillary thyroid carcinomas. Oncogene 2004;23:5958-5967.

23 He X, Wei Q, Zhang X, et al. Immunohistochemical expression of CXCR4 in thyroid carcinomas and thyroid benign lesions. Pathol Res Pract 2010; 206:712-715.

24 Melillo RM, Castellone MD, Guarino V, et al. The RET/ PTC-RAS-BRAF linear signaling cascade mediates the motile and mitogenic phenotype of thyroid cancer cells. J Clin Invest 2005;115:1068-1081.

25 Gonzalez HE, Leiva A, Tobar H, et al. Altered chemokine receptor expression in papillary thyroid cancer. Thyroid 2009;19:957-965.

26 Wagner PL, Moo TA, Arora N, et al. The chemokine receptors CXCR4 and CCR7 are associated with tumor size and pathologic indicators of tumor aggressiveness in papillary thyroid carcinoma. Ann Surg Oncol 2008;15:2833-2841.

27 Yasuoka H, Kodama R, Hirokawa M, et al. CXCR4 expression in papillary thyroid carcinoma: induction by nitric oxide and correlation with lymph node metastasis. BMC Cancer 2008;8:274. 